\title{
Basic Architecture and Principle Analysis of Farmland's Automatic Irrigative System
}

\author{
Li Rongzheng $^{1}$ and Zhu Jieying ${ }^{2}$ \\ Information Center, Shanghai University of Engineering Science, Shanghai 201620, \\ China \\ lrz@sues.edu.cn,zjy@sues.edu.cn
}

\begin{abstract}
It proposes a basic architecture of farmland's irrigative system based on the rapid development of Internet technology which can be applied to the modern management of farmland in this paper, and it introduces the component unit in detail, and analyz deeply in principle. System uploads data of farmland's environment temperature and humidity to Internet by remote wireless. Through computer terminals' datc analysis and field controlling irrigation valve by remote wireless, it implements the intelligent of the farmland's management.
\end{abstract}

Keywords: Automatic irrigation, wireless sensor network (WSN), ZigBee, Internet

\section{Introduction}

Since 21 st century, information technorogy revolution has brought earth-shaking changes to each industry with rapid development of modem information technology. The system is an application try of Internet technology that is used in farmland management, and it has important significance regardless of the promotion of the Internet technology or to the development of modern agriculture. It uses the latest close wireless ZigBee technology, forms a wireless sensor network (W/SN), real-time collects crops growth environment information, judges and analyzes the environment for the growth of crops, and decides irrigative model to provide a comfortable environtment foy growth of crops. The technical scheme not only can improve the yield of erops, but also can greatly save water resources to implement large tracts farmland management mores scientific and effective. In today's advocacy of energy conservation and emissions reduction, it meets the need of cycle of the social development, reduce agricultural dependence on human for a long time at the same time, and it will promote modern agriculture development in the direction of intelligent and scientific. This innovation will bring new atmosphere to development of modern agriculture, and will accelerate goticultural modernization realization.

\section{System's Composition}

This paper proposes an automatic farmland irrigation system which through building close Zigbee wireless sensor network (WSN), acquisition of farmland environment temperature and humidity, send data of temperature and humidity what was collected through DTU remote wireless transmission to Internet. Computer's terminal receives temperature and humidity of farmland environment, and through comparative analysis of the default value as well as historical data default values, to judge whether to irrigation, determine what kind of pattern, forming the corresponding instruction returned to DTU. DTU sends instructions to microprocessor, and microprocessor executes programs according to the received instructions, so as to realize the automatic irrigation of farmland. Users can access to the Internet computer terminals at any time to check the latest information and irrigation of farmland environment. Farmland irrigation system is mainly composed of data acquisition unit, control unit, remote 
wireless communication unit, remote monitoring unit, power supply management unit, and system general structure diagram is shown in Figure 1.

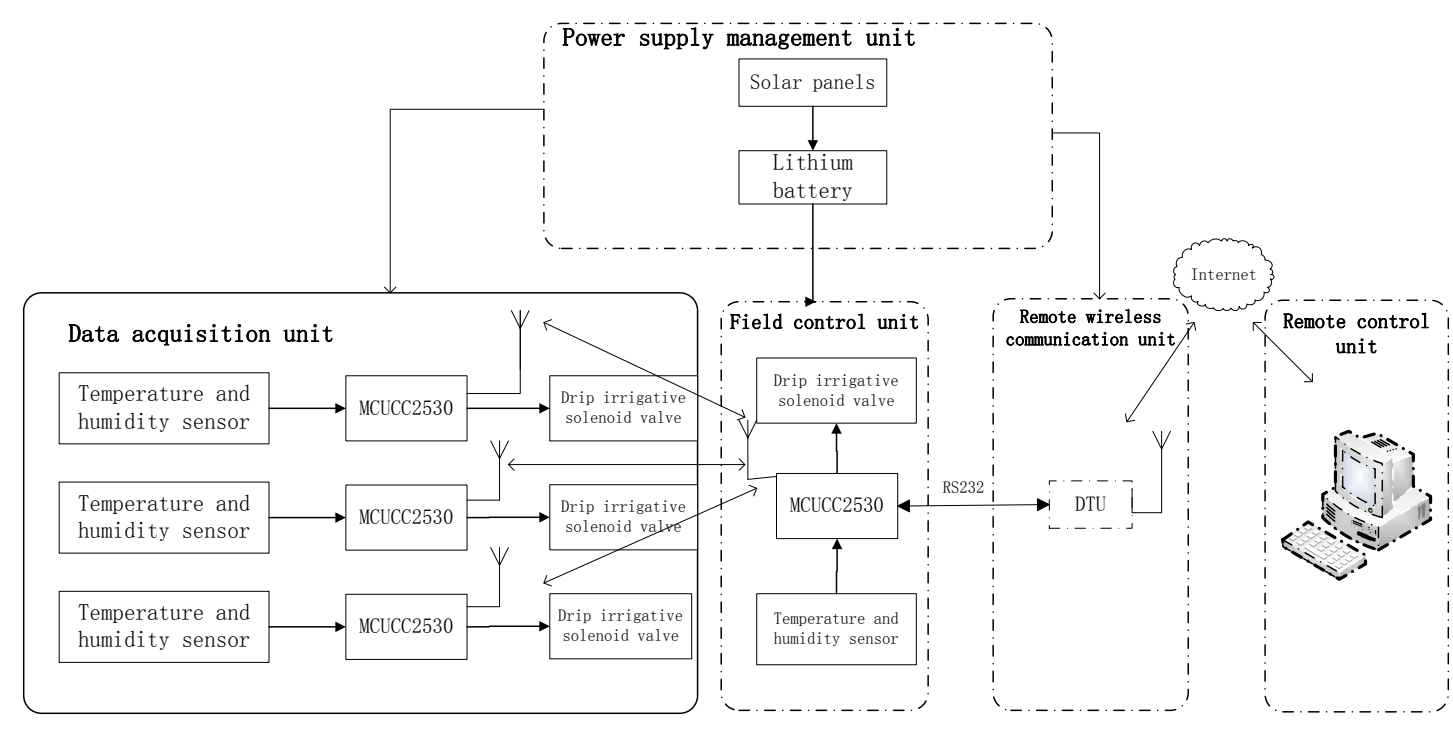

Figure 1. System's Block Diagram

Data acquisition unit is essentially a wireless sensor network (WSN) which uses the latest ZigBee network technology. ZigBee technology is a kind of wireless communication protocol which is based on IEEE 802. 15. 4. Its charactetistic is low cost, low power consumption, low complexity, high reliability, two-way transmission, qexible networking, and it is a new wireless network technology that is short distance, low rate. It has Large capacity of the network nodes, and it can suppott more than 60000 nodes in theory, and it can form a variety of topology in the form of network between hodes, it is shown in Figure 2. It chooses the simpler star topology structure, data acâuisition unit collects sensor data of temperature and humidity, sends measured information to coordinator unifying, sends via wireless to the scene the microprocessor con trol unit.

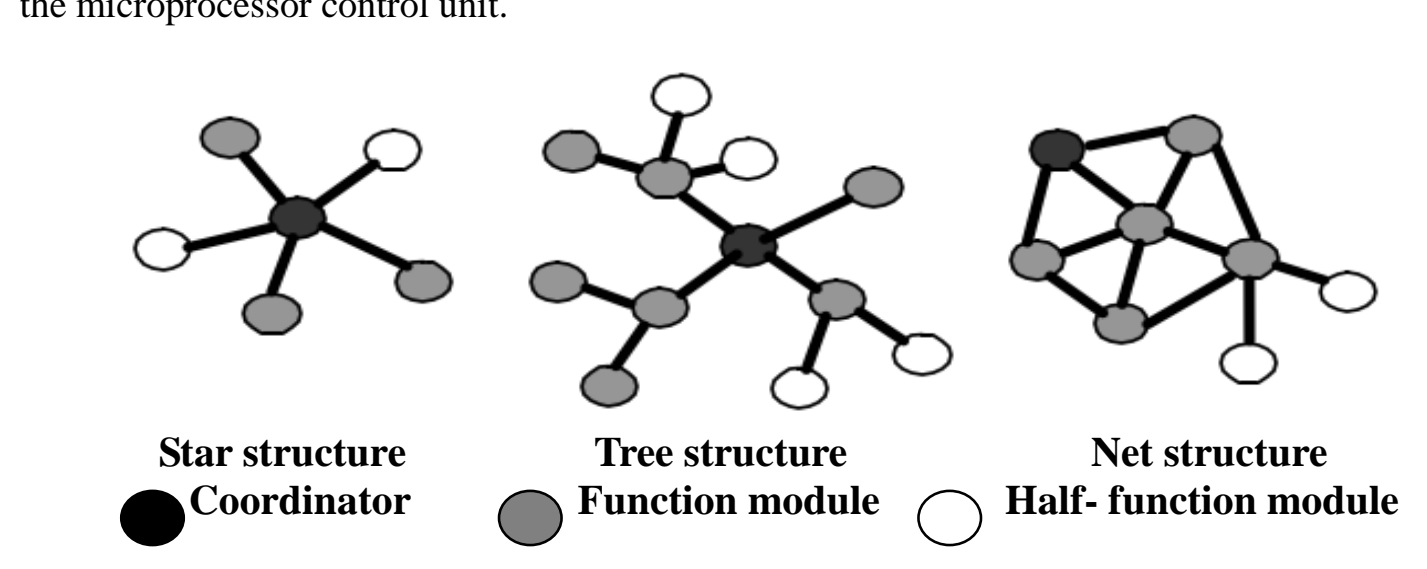

Figure 2. ZigBee's Network Topology

Field control unit is responsible for the entire scene ZigBee network operation maintenance. It gathers all data of temperature and humidity, frames temperature's and humidity's data of scene node, sends to remote wireless communication unit, and receive instruction from remote wireless communication unit, such as controlling the scene of some irrigation electromagnetic valve opening and closing.

Remote wireless communication unit is responsible for posting temperature's and humidity's data to Internet through the remote wireless network operators according to the destination IP and port number. At the same time the data that is received from the Internet will transmit to field control unit, and the data transmission process is completely transparent. 
Remote monitoring unit is responsible for the receiving, storage, display and analysis of temperature's and humidity's data, and power supply management unit mainly provides power supply for each unit at the scene.

\section{Hardware Design}

System's hardware design mainly includes data acquisition unit, field control unit remote wireless communication unit and power management unit.

\subsection{Hardware Design of Data Acquisition Unit}

Data acquisition unit hardware circuit is mainly composed of CC2530 wireless single chip microcomputer minimum system, soil temperature and humidity sensor SHT10, irrigation of solenoid valve and drive circuit. CC2530 is a close wireless single chip microcomputer that is compatible with ZigBee protocol developed by TI company, and CPU is enhanced 8051 kernel which works single clock instruction cycle. ZigBee has not only its supefror wireless function, but also it has powerful peripherals. It contains 18 interrupt source, $12 \mathrm{AD}$ sampling, 4 timers, a sleeping timer that can work in ultra low power mode. It can reduce system's power consumption to minimize, and it also includês two wây USART serial communication that can be configured to asynchronous UART or synchronous SPI communication mode.

The Specific chart that is MCU CC2530, temperature and hunidity sensor SHT10, electromagnetic valve and its drive circuit is shown in Figure 3. Each chip power foot should join the appropriate decoupling capacitor to Ceduce power noise that caused by external electromagnetic interference.

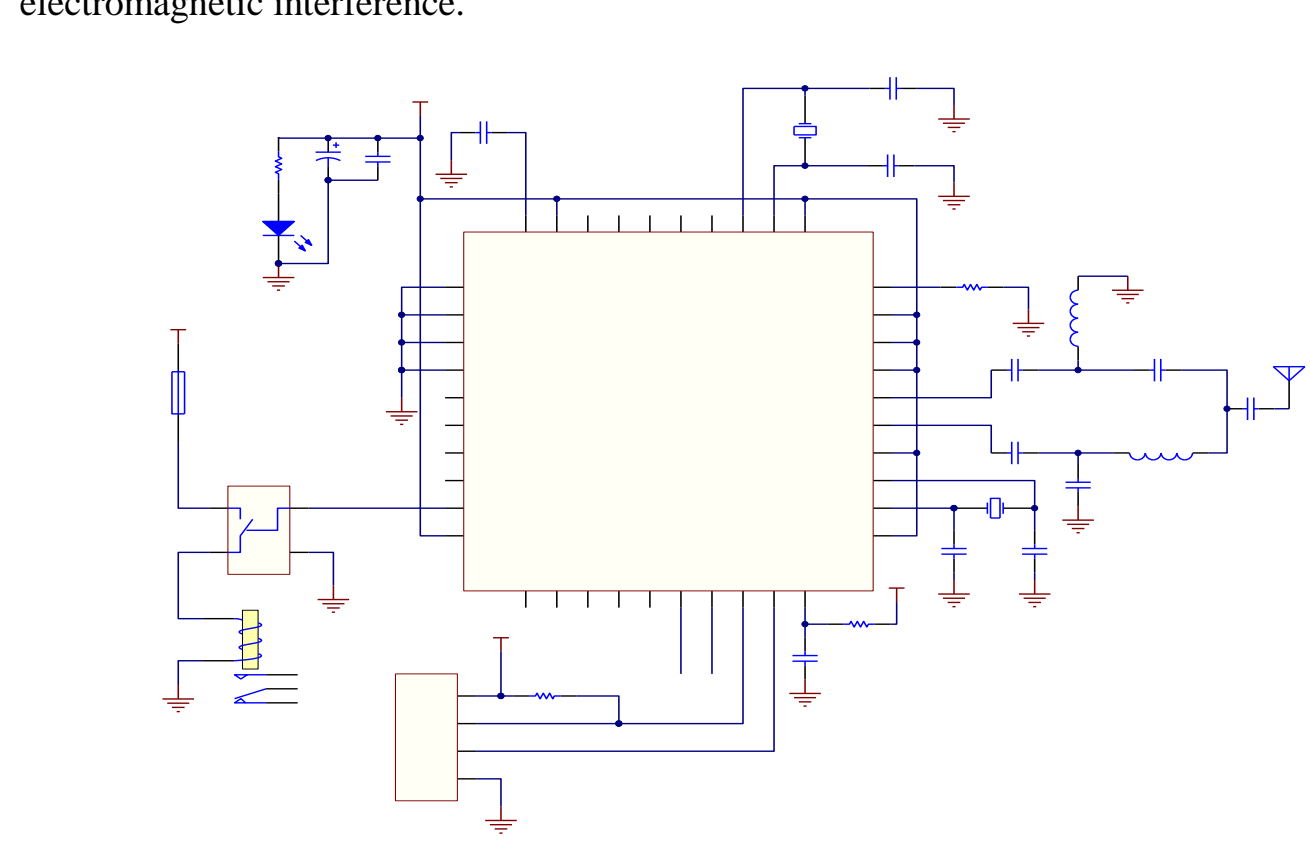

Figure 3. Principle Diagram of Data Acquisition Unit

Hardware design of field control unit is basically same as data acquisition unit, the difference is that field control unit should connect with remote wireless communication unit.

\subsection{Hardware Design of Remote Wireless Communication Unit}

Remote wireless communication unit hardware mainly includes DTU and level conversion circuit. DTU is terminal of wireless data transmission, and it provides users with wireless long-distance data transmission function by public network. The system uses H7710 DTU, supplies wide voltage $5 \mathrm{~V}$ to $36 \mathrm{~V}$ dc power, supports sleeping function, and it is compatible 
with data interface RS232 protocol. It can be connected to a serial port equipment directly to realize data transparent transmission function. Because DTU communication interface is a standard RS232 protocol level, it must make microprocessor of UART communication level convert to standard RS232 protocol level to realize of communication with DTU. The specific circuit principle as shown in Figure 4.

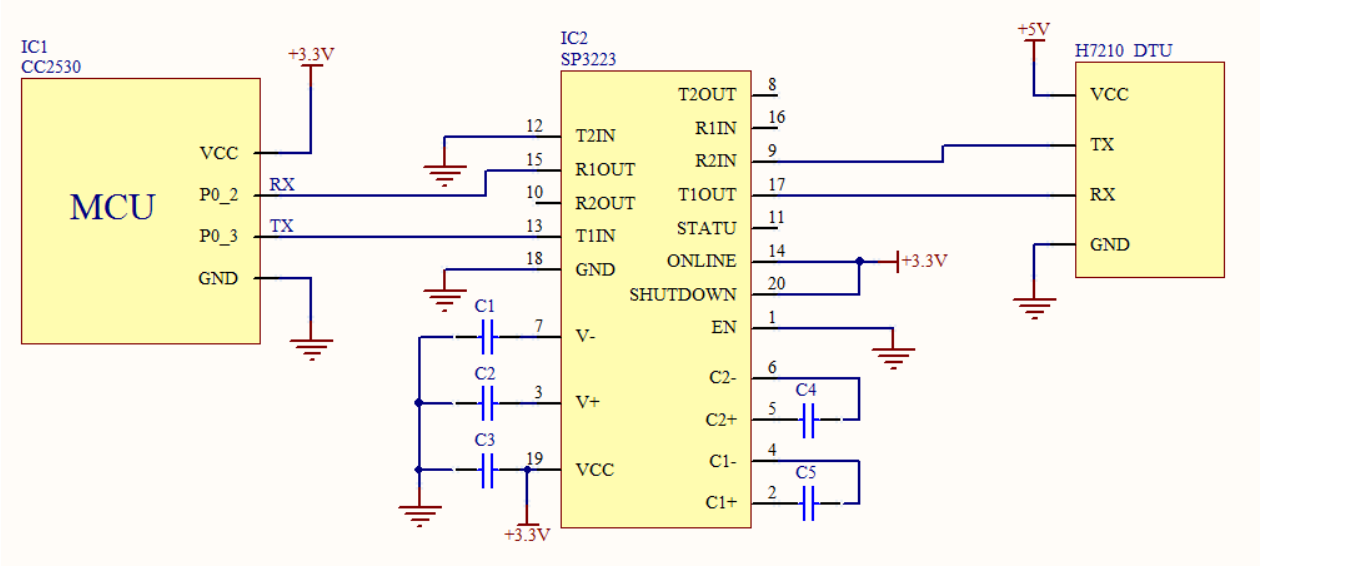

Figure 4. RS232 Level Conversion Circuit

\subsection{Hardware Design of Power Supply Management Unit}

Power supply management unit is mainl responsible for providing power supply to field control unit to ensure power supply persistence whin uses large capacity lithium battery supply of energy. Because battery voltage 1 s not suítable for supplying power directly, it must transformative battery voltage to needed voltage grade accordingly. In order to improve efficiency of power supply at the same time, ifadopts switching power supply technology to realize battery voltage transfarmation, and it converts for microprocessor, remote communication unit and the power supply voltage of solenoid valve need. To meet the needs of vast farmland irrigation automation and strengthen effective management and use of battery, it also provides solar panels charge interface to access to corresponding voltage grade of solar panels. In the case of sunny, it can realize for lithium battery power supply and extend greatly prolong life time of battery. System also join battery voltage monitoring function that microprocessofiner can sample battery alarm signal when battery power needs to be added.

\section{Software Design}

System's software mainly includes data acquisition unit, field control unit and remote monitoring unit. System's software design always aims at reliability, practicability and extensibility, especially in order to ensure reliable work of the whole system. Software introduces watching-dog function to reset automatically after judging system's operation is not normal. In order to ensure reliable data communication in system, it formulates protocols and standards of relevant data communication to find problems and try to repair timely when communication is not normal; in order to decrease inherent power of system's components, it uses dormancy mechanism that it can collect sensor's data at every interval time and the rest of the time the system is enter to a dormant state to reduces system's power consumption greatly.

\subsection{Software Design of Data Acquisition Unit}

According to field instructions of controller giving, it should perform corresponding operation procedure. Data acquisition unit's function is to realize timing acquisition of temperature's and humidity's data, and once it get data collection command by 
microprocessor unit accessing SHT10, it will read temperature's and humidity's data to uploaded them to field control unit microprocessor automatically. If it is sleeping command, it will be into dormancy directly to reduce system's power consumption; and if it is irrigation command, it will open electromagnetic valve and timer to close valve when after irrigation time.

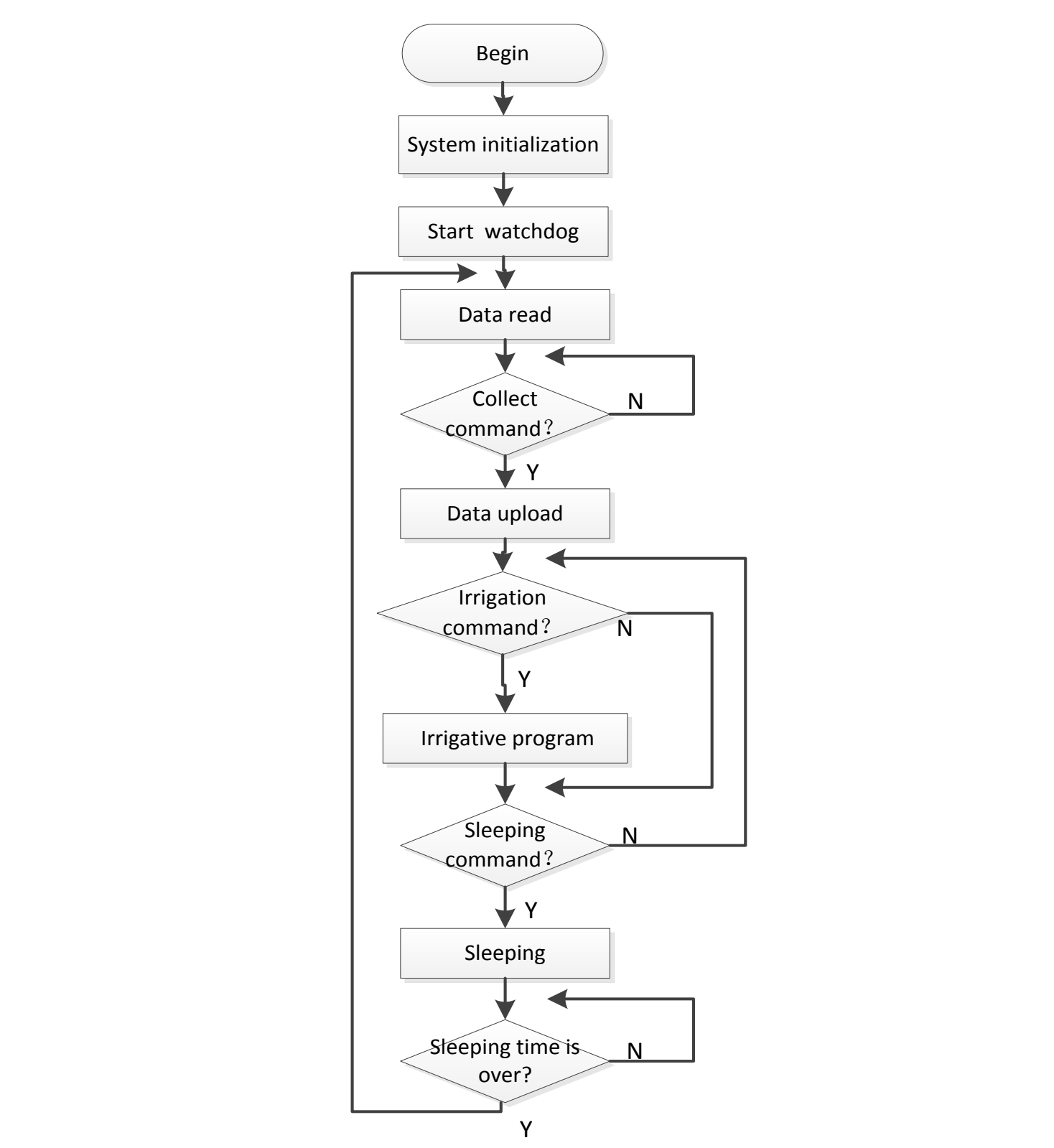

Figure 5. Flow Chart of Data Acquisition Unit's Software

\subsection{Software Design of Control Unit}

Field control unit mainly completes field data collection and send the data to DTU, and it realize to remote sent data through DTU. It is also receive DTU's returned data, and it realize remote control of the scene according to executing corresponding operation from return instruction. Field wireless communication is always initiated by field microprocessor control unit, by way of naming, and it polls data acquisition unit of each microprocessor to avoid conflicts of wireless communications effectively. 
Figure 6. Flove Chart Field of Control Unit Software

\subsection{Software of Remote Monitoring Unit}

According to datacommunication protocol, remote monitoring unit realizes data remote receiving, unpacking storing, displaying and analysis. Through data analysis and program setting, it completes corresponding operations, such as whether to open valve for irrigation, whether there is a warning message (a sensor doesn't work properly or lithium battery needs to charge) nis displays data on the computer terminals, and it can also form the history curve according to users' requirements to provide data for researching and analysis crop growth condition. Remote monitoring unit can also deploy, delete, and add a node for field ZigBee's oommunication network, and it can set sampling interval at the same time.

\section{Conclusion}

This paper uses advanced ZigBee's technology, it forms a wireless temperature and humidity sensor network from architecture theory. It sets up a basic Internet model, and it realizes remote monitoring of farmland's environmental temperature and humidity. Through computer terminal's data analysis and control, it realizes opening and closing of irrigation's valve, and it achieves the expected goal of system. As long as there has network, it can real-time monitor farmland environment at any time, and the system will also automatic drip irrigation according to collected environmental data to ensure that crops growing in a comfortable environment. It realizes vast fields of intelligent management really, and the 
application of this system should promote agricultural development in the more modern direction.

At the same time, if forming ZigBee nodes into more complex mesh network, it can realize remote monitoring of vast farmland. The system also has strongly portability, and it can be very convenient portable to other needs of real-time monitoring and automatic control industry.

\section{References}

[1] C. Haiming, C. Li and X. Bin, "Comparative and Study on Internet's architecture and implementation method", Journal of computers, vol. 4, no. 1, (2013).

[2] CC253X User's Guide [Rev.A]. [2010-06-25]. http://focus.ti. com/ docs/ prod/ folders/ print/- cc2530.html.

[3] ZigBee Alliance, ZigBee Specification 2006. [2010-08]. http://www.zigbee.org

[4] Z. Yongfeng, "ZigBee's wireless sensor network (WSN)", Beijing: Beijing university of posts and telecommunications publishing (2011).

[5] Y. Shuanke, “Analog electronic technology foundation", Beijing: Higher education press (2010)

[6] L. Jie, "Tutorial of Protel DXP circuit design (second edition)", Beijing: Tsinghua university press (2010).

[7] C. Danhua, "Digital electronic technology foundation", Beijing: Electronic industry press (2011)

[8] Z. Quan and T. Weiqing, "Implementation of embedded TCP/IP protocol stack based on GPRS wireless communication, Industrial control computer, vol. 21, no. 05, (2008)

[9] IEEE 802.15.4-2003Std: Wireless Medium Access Control (MAC) and hysical Layer (PHY) Specifications for Low-Rate Wireless Personal Area Network (LR-WPANs). [2010-08]. http:/Awww.ieee802.org/

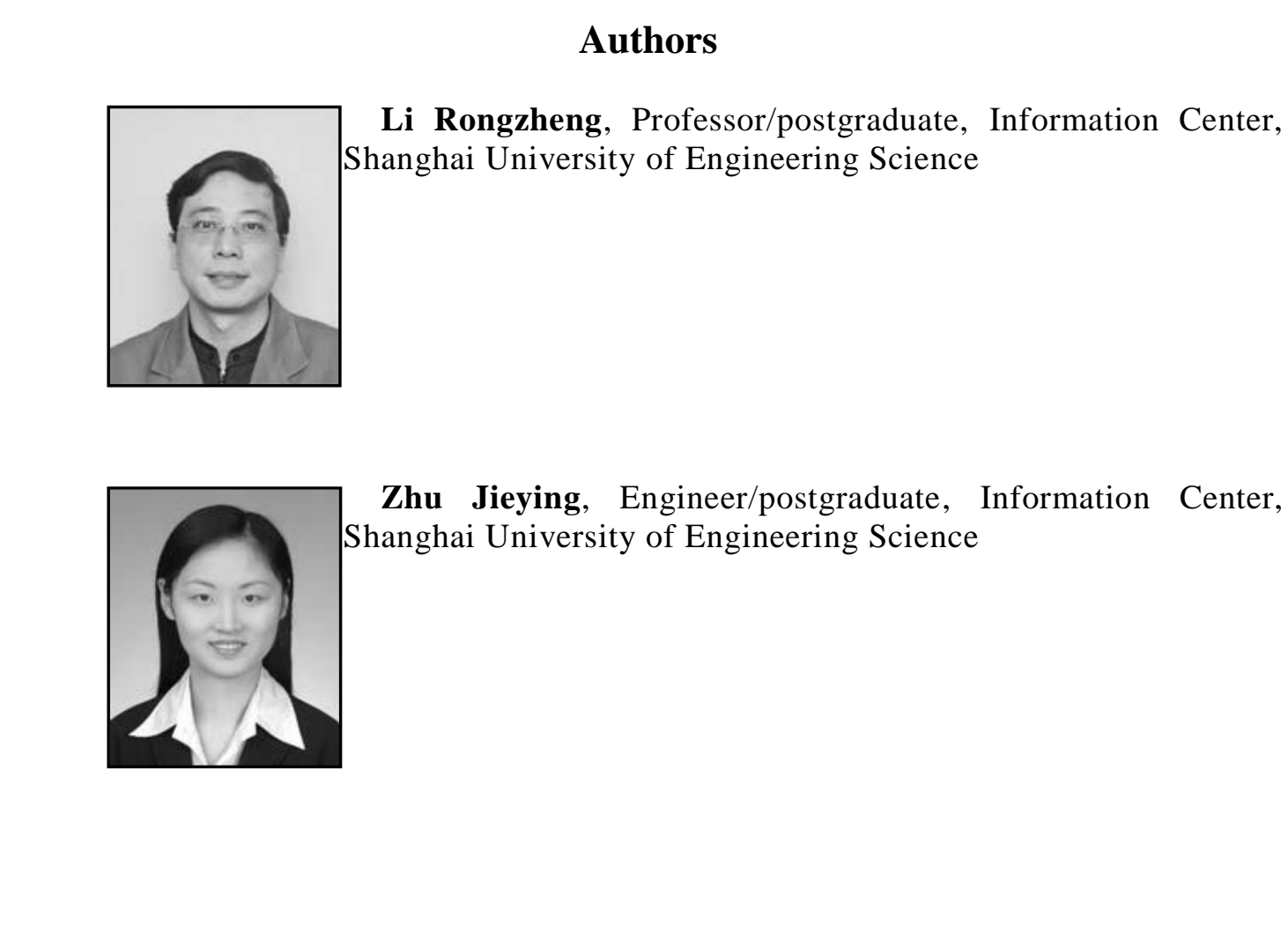




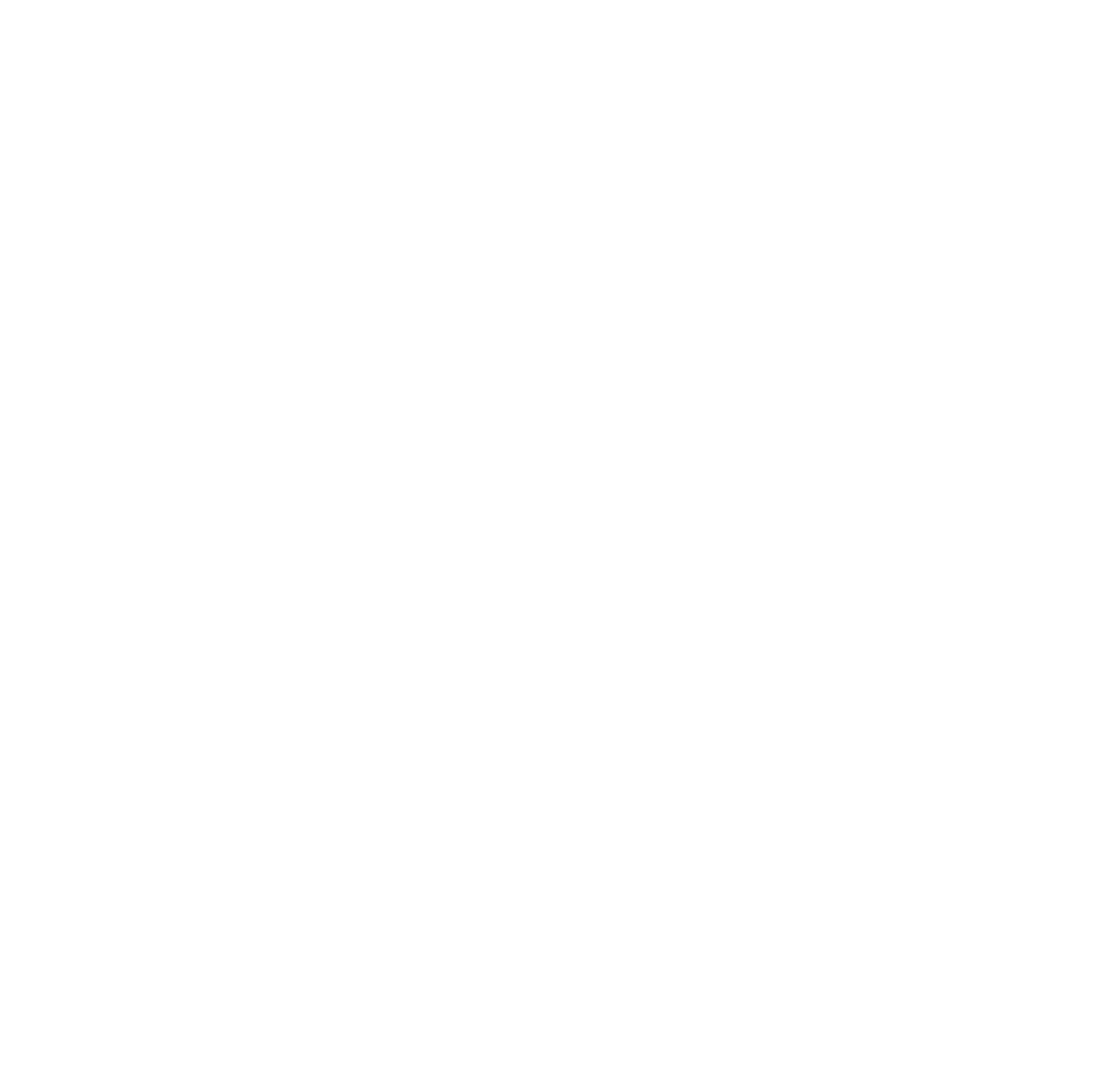

